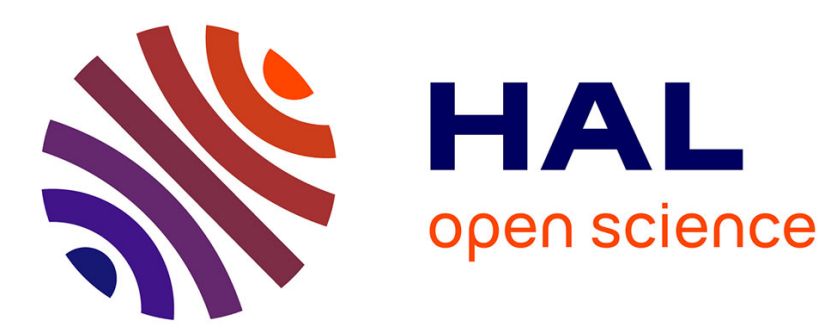

\title{
Reactive Leidenfrost droplets
}

Christophe Raufaste, Yann Bouret, Franck Celestini

\section{To cite this version:}

Christophe Raufaste, Yann Bouret, Franck Celestini. Reactive Leidenfrost droplets. EPL - Europhysics Letters, 2016, 114, pp.46005. 10.1209/0295-5075/114/46005 . hal-01358084

\section{HAL Id: hal-01358084 https://hal.science/hal-01358084}

Submitted on 6 Feb 2022

HAL is a multi-disciplinary open access archive for the deposit and dissemination of scientific research documents, whether they are published or not. The documents may come from teaching and research institutions in France or abroad, or from public or private research centers.
L'archive ouverte pluridisciplinaire HAL, est destinée au dépôt et à la diffusion de documents scientifiques de niveau recherche, publiés ou non, émanant des établissements d'enseignement et de recherche français ou étrangers, des laboratoires publics ou privés. 


\title{
Reactive Leidenfrost droplets
}

\author{
C. Raufaste ${ }^{1}$, Y. Bouret $^{1}$ and F. Celestini ${ }^{1}$ \\ 1 Université Nice Sophia Antipolis, CNRS, LPMC, UMR 7336, Parc Valrose, 06100 Nice, France
}

\begin{abstract}
PACS 68.03.Cd - Surface tension and related phenomena PACS 68.03.Fg - Evaporation and condensation of liquids PACS 47.55.D- - Drops and bubbles
\end{abstract}

\begin{abstract}
We experimentally investigate the reactivity of Leidenfrost droplets with their supporting substrates. Several organic liquids are put into contact with a copper substrate heated above their Leidenfrost temperature. As the liquid evaporates, the gaseous flow cleans the superficial copper oxide formed at the substrate surface and the reaction maintains a native copper spot below the evaporating droplet. The copper spot can reach several times the droplet size for the most reactive organic compounds. This study shows an interesting coupling between the physics of the Leidenfrost effect and the mechanics of reactive flows. Different applications are proposed such as drop motion tracking and vapor flow monitoring.
\end{abstract}

In spite of its discovery more than two centuries ago [1], the Leidenfrost effect is still the subject of numerous applied and fundamental researches. One can note the recent studies concerning the effect of an electric [2] or a magnetic field [3] on theses droplets and the studies concerning the directed motion on structured surfaces $[4,5]$. The unexpected take-off of sub-millimetric Leidenfrost droplets has been also examined [6] and different theoretical analysis are now proposed to understand this phenomenon $[7,8]$.

Linking the Leidenfrost effect and chemistry has recently brought out the possibility to use droplets in calefaction as micro-reactors giving rise to a new nanofabrication method [9]. Already in his seminal paper [10], Boutigny dedicated a whole chapter to the specific chemical properties of Leidenfrost droplets. He made experiments with acid droplets that react strongly with metallic substrates. While he expected an increase of the oxidation rate as the temperature was increased, the reactivity suddenly dropped when the temperature reached the Leidenfrost temperature of the acid due to the vanishing contact between the acid and the substrate.

In this letter we present a first attempt to induce a chemical interaction between a Leidenfrost droplet and the substrate on which it is standing. We experimentally show that the vapor escaping from a Leidenfrost droplet can react with the supporting substrate. To summarize, above a critical temperature, the vapor flow acts as a reducing agent and reacts with the copper substrate initially superficially oxidized. This leads to the formation of a native copper spot that persists as long as the droplet is not evaporated. This effect was observed for several organic liquids and the critical reaction temperatures are measured. A redox mechanism is proposed and the observations are related to the reducing properties of the liquid. The spot size is comparable to the one of the droplet but can become very large with chemical compounds such as ethylene glycol. Finally, applications are given such as the surface cleaning, the tracking of a droplet on an inclined substrate and the monitoring of the vapor flow on a textured surface.

Experiments. -

The experimental set-up consists of a hot plate on which a copper substrate is deposited. The copper substrate is sandblasted before any experiment to ensure an homogeneous surface roughness. The substrate temperature is measured and controlled through a PT100 temperature sensor coupled to the heating system. The temperature in our range $\left(200-500^{\circ} \mathrm{C}\right)$ is sufficiently high for an oxide copper film to form on the native copper substrate. For instance, it takes a few minutes for the oxide to form on a copper substrate heated at $T=380^{\circ} \mathrm{C}$. Images are taken using a high speed camera at a frame rate standing between 5 and 1000 frames per second. Drops are deposited on the substrate and they levitate on their own vapor. They are maintained in place through the contact with a small capillary (Fig. 1).

Several organic liquids are tested: ethanol, isobutanol, isopropyl alcohol, tert-butyl alcohol, ethylene glycol and 


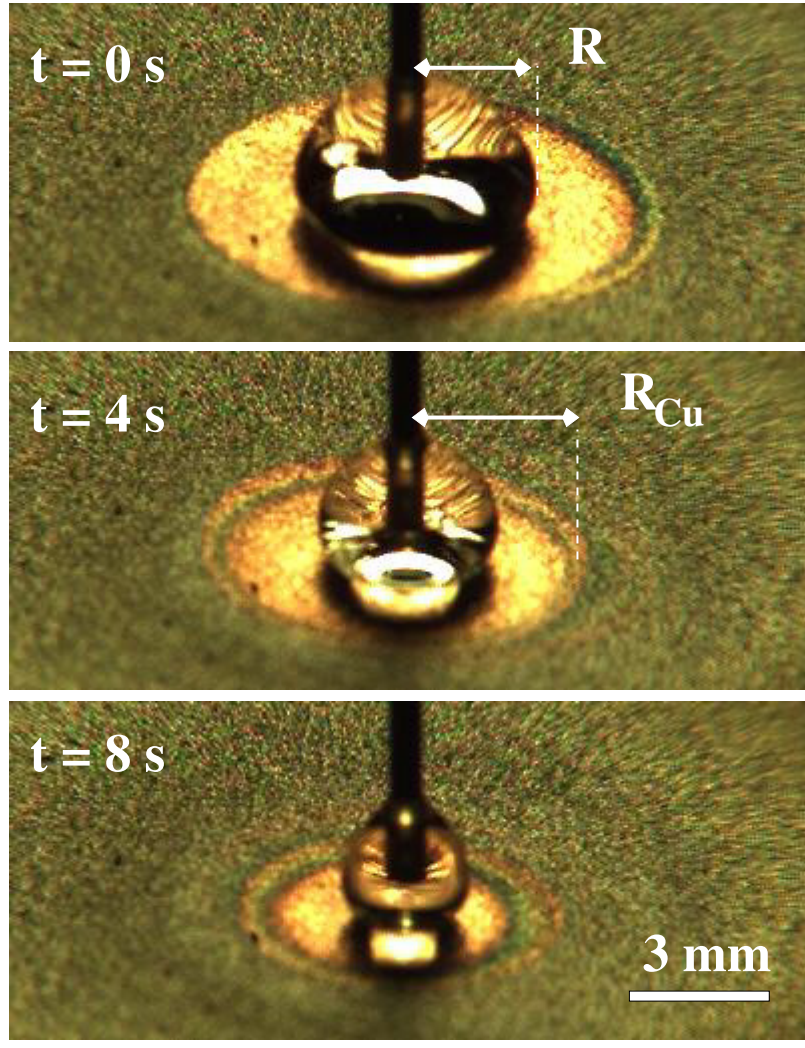

Fig. 1: (Color on-line) Evaporation of an ethanol Leidenfrost droplet of radius $R$ standing on a copper substate. The substrate is initially oxidized and appears dark. The radius of the native copper spot below the droplet is denoted $R_{\mathrm{Cu}}$. acetone. For almost all of them, from the Leidenfrost temperature to a higher critical temperature the droplet levitates on the substrate without any visible effect on the substrate. Above this critical temperature, a reaction is observed and a spot of native copper of radius $R_{\mathrm{Cu}}$ appears below the droplet of radius $R$ (Fig. 1). We note that the reaction is different from a complete copper-catalyzed oxidation [11]: it does not emit any radiation in the visible range and is not inhibited by working under oxygen-free conditions (nitrogen atmosphere). As expected due to the droplet evaporation, $R$ decreases with time (insert of Fig. 2 ). We observe that $R_{\mathrm{Cu}}$ decreases too. This suggests that $R_{\mathrm{Cu}}$ results from an equilibrium between the copper oxidation rate occurring naturally and the copper oxide reduction rate due to the reactive Leidenfrost vapor flow. Colored fringes found in the vicinity of the native copper spot give an indication about the copper oxide film thickness resulting from the equilibrium between the two reactions (Figs. 1 and 4). Once the droplet is totally evaporated, the native copper spot disappears and the superficial copper oxide layer covers all the substrate again. Working under oxygen-free conditions prevents the substrate re-oxidation and maintains the native copper aspect where the reaction occurred. This point is illustrated in one movie given in

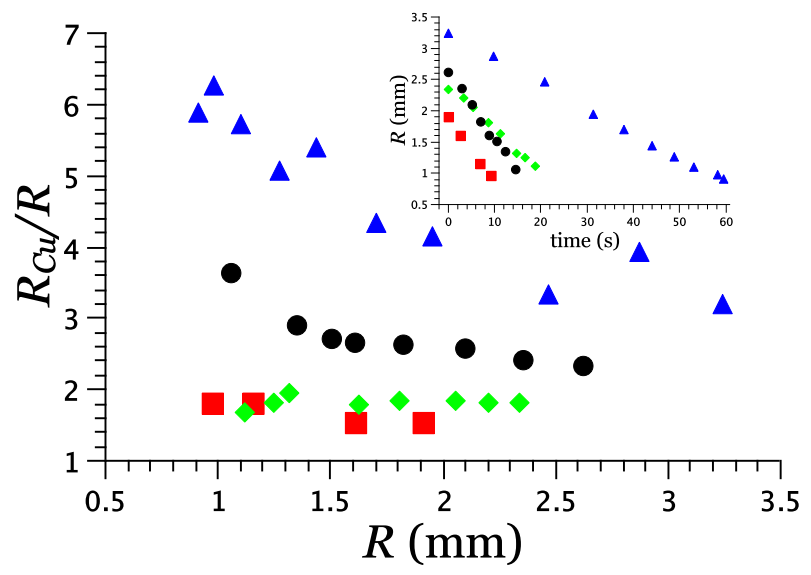

Fig. 2: (Color on-line) Ratio $R_{\mathrm{Cu}} / R$ as a function of $R$ for droplets of ethanol (green diamonds), isobutanol (red squares), isopropyl alcohol (black dots) and ethylene glycol (blue triangles). The temperature of the substate is fixed at $380^{\circ} \mathrm{C}$. Insert: $R$ as a function of time for the same dataset. The origin of time is taken arbitrarily as the first measurement time.

the supplementary material (Movie1.avi): under nitrogen atmosphere a cross is drawn by guiding an ethanol drop with the capillary. The cross remains as long as the nitrogen flux is maintained. When the latter is removed, the substrate is re-oxidized and the colored fringes are momentarily observed before the cross totally disappears.

To quantify the phenomenon we measure both $R$ and $R_{\mathrm{Cu}}$ as a function of time (insert of Fig. 2) for experiments performed on different liquids and for a substrate heated at a fixed temperature $T=380^{\circ} \mathrm{C}$. We plot in Fig. $2 R_{\mathrm{Cu}} / R$ as a function of $R$. The value of this ratio strongly depends on the liquid used. The larger ratios are obtained for ethylene glycol (Fig. 3) while the lower values are observed for ethanol and isobutanol droplets. For two liquids (ethylene glycol and isopropyl alcohol) the ratio $R_{\mathrm{Cu}} / R$ is a decreasing function of $R$ while for the two other liquids (ethanol and isobutanol) this ratio seems to be quite constant.

The critical reaction temperature $T_{c}$ defined above strongly depends on the liquid. To measure $T_{c}$ we slowly increase the temperature of the substrate until the reaction is observed. During this procedure droplets of the same size, roughly $1 \mathrm{~mm}$ in radius, are deposited on the substrate. The critical temperature measurements are reported in Table 1. $T_{c}$ is always smaller than $300^{\circ} \mathrm{C}$ for the primary and secondary alcohols : we found between 200 and $250^{\circ} \mathrm{C}$ for ethanol, isobutanol and isopropyl alcohol, and the critical temperature for the ethylene glycol is smaller than $250^{\circ} \mathrm{C}$ and could not be measured since the Leidenfrost effect begin to disappear below this temperature. For the tert-butyl alcohol and the acetone, the reaction initiates at larger temperatures and develops over a temperature range $\left[T_{c}^{\min } T_{c}^{\max }\right]$. At $T_{c}^{\text {min }}$, the reaction is 


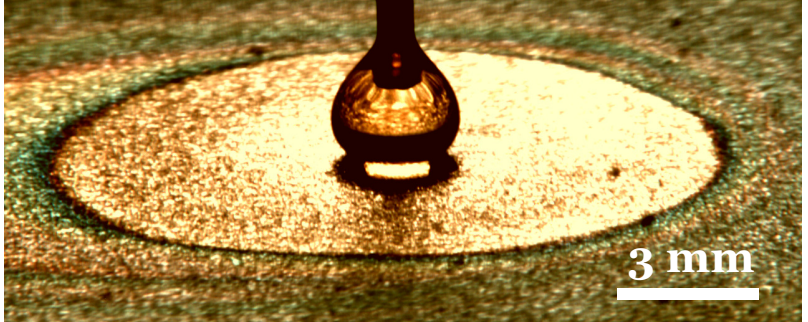

Fig. 3: (Color on-line) Evaporation of an ethylene glycol Leidenfrost droplet.

initiated but only a tiny spot is observed that corresponds to the colored fringes of the oxide film. Only for the temperatures larger than $T_{c}^{\max }$ the reaction is strong enough for the native copper of the substrate to be observed.

\section{Redox mechanism. -}

We propose a heterogeneous redox mechanism to account for the chemical reaction. Copper oxides form redox couples with native copper (redox potentials $E^{0}\left(C u^{+} / C u_{(s)}\right)=+0.52 \mathrm{~V}$ and $E^{0}\left(C u^{2+} / C u_{(s)}\right)=$ $+0.34 \mathrm{~V})$, while primary and secondary alcohols are reducing agents with redox potentials around $-0.20 \mathrm{~V}$ in standard conditions. The copper oxides-alcohols reactions are thermodynamically favored (but slow) at $25^{\circ} \mathrm{C}$ and we expect a larger reaction rate for increasing temperatures, which we observe.

Modeling the ratio $R_{C u} / R$ and the different values measured between the several chemical compounds is beyond the scope of the article. This is not obvious because it would require to precisely describe the exact shape of the flow field around the droplet and its chemical composition, a difficult task due to the mixing of the reactive vapor with the surrounding air. We have measured the evaporation rates for the alcohols analyzed in Fig. 2 (Tab. 1). We noticed that the largest copper spots were obtained for the least volatile chemical compound. This suggests that the reactivity with the substrate is probably the important parameter to understand the differences between the several organic species.

Inorganic redox species. Metal oxides may be used as oxygen carriers for the controlled redox reactions of simple organic species [11] to their possibility of chemical looping combustion with a view to achieve the sequestration of the produced carbon dioxide (see $[12,13]$ for a review and current developments). Actually, the copper surface is a mix of copper(I) oxide $\left(\mathrm{Cu}_{2} \mathrm{O}\right)$ and copper(II) oxide $(\mathrm{CuO})$ that can produce some adsorbed oxygen species as:

$$
\mathrm{Cu}_{x} \mathrm{O} \rightleftharpoons x \mathrm{Cu}+\frac{1}{2} \mathrm{O}_{2}, \quad 1 \leq x \leq 2 .
$$

Oxidizing the organic species. None of our tested compounds may carry out a direct spontaneous oxidation by gaseous $\mathrm{O}_{2}$. So, in all cases, we consider only the first possible reaction of the species by the adsorbed oxygen, since we expect the partial pressure of the formed species to vanish under the action of the natural convection. After that first reaction, a layer of copper is formed on the surface.

For a primary alcohol $\mathrm{R}_{1}-\mathrm{CH}_{2}-\mathrm{OH}\left(\mathrm{R}_{1}=\mathrm{CH}_{3}\right.$ : ethanol, $\mathrm{R}_{1}=\left(\mathrm{CH}_{3}\right)_{2}-\mathrm{CH}$ : isobutanol, $\mathrm{R}_{1}=\mathrm{HO}-\mathrm{CH}_{2}-$ : ethylene glycol), we have the formation of an aldehyde function as

$$
\mathrm{R}_{1}-\mathrm{CH}_{2}-\mathrm{OH}+\mathrm{Cu}_{x} \mathrm{O} \rightarrow x \mathrm{Cu}+\mathrm{R}_{1}-\mathrm{COH}+\mathrm{H}_{2} \mathrm{O} .
$$

The loss of one molecule of water make this reaction very irreversible.

Analogously, for a secondary alcohol $\mathrm{R}_{1}^{\prime}-(\mathrm{CHOH})-\mathrm{R}_{2}^{\prime}$ $\left(\mathrm{R}_{1}^{\prime}=\mathrm{R}_{2}^{\prime}=\mathrm{CH}_{3}\right.$ : isopropyl alcohol), we have the corresponding formation of a ketone function as

$$
\mathrm{R}_{1}^{\prime}-(\mathrm{CHOH})-\mathrm{R}_{2}^{\prime}+\mathrm{Cu}_{x} \mathrm{O} \rightarrow x \mathrm{Cu}+\mathrm{R}_{1}^{\prime}-\mathrm{CO}-\mathrm{R}_{2}^{\prime}+\mathrm{H}_{2} \mathrm{O} .
$$

Unfortunately, the produced quantities are too small to allow specific characterizations, and devising a setup to trap the emitted vapour is far beyond the scope of this article. Moreover all the classical tests for carbonyl compounds would not stand the high temperature copper vicinity. For terbutanol and acetone, an oxidation occurred but at much higher temperature, with indicates that some minor or major organic skeleton changes are involved. Indeed, we could think of a possible dehydration/oxidation of terbutanol into epoxy-isobutylene, or the oxidation of the acetone à la Bayer-Villiger into methyl-ethyloate. Nonetheless, the temperature increase is quite significant, which indicates that the following generic oxidative degradation occurs

$$
\left\{\begin{array}{l}
\mathrm{C}_{n+m} \mathrm{H}_{y} \mathrm{O}_{z}+p \mathrm{Cu}_{x} \mathrm{O} \rightarrow n \mathrm{CO}_{2}+m \mathrm{CO}+\frac{y}{2} \mathrm{H}_{2} \mathrm{O}+p x \mathrm{Cu} \\
p=2 n+m+\frac{y}{2}-z
\end{array} .\right.
$$

Copper oxides regeneration. If some dioxygen is available, the fresh copper layer shall be readily oxidized by the inverse reaction of (1)

$$
x \mathrm{Cu}+\frac{1}{2} \mathrm{O}_{2} \rightleftarrows \mathrm{Cu}_{x} \mathrm{O} .
$$

For usual metal or metal-oxide catalyzed reactions we expect the released enthalpy to overheat the solid substrate (up to a glowing state), but here the heat is dissipated by convection. Inversely, if no oxygen is present, then the copper should remain visible, up to its diffusion inside the oxide layer.

\section{Applications. -}

As seen previously, the reactive Leidenfrost effect emphasizes a simple way to clean locally, at the drop scale, the surface of a substrate (Movie1.avi in the supplementary material). This effect might be used to track droplet trajectories as well. As an example we followed the motion of an ethanol droplet on an inclined copper substrate 


\begin{tabular}{|c|c|c|c|}
\hline Chemical compound & Leidenfrost $\mathrm{T}\left({ }^{\circ} \mathrm{C}\right)$ & $T_{c}\left({ }^{\circ} \mathrm{C}\right)$ & Evaporation rate $(\mathrm{mol} / \mathrm{s})$ \\
\hline ethanol $\mathrm{CH}_{3} \mathrm{CH}_{2} \mathrm{OH}(\mathrm{I})$ & $136 \pm 5$ & $245 \pm 5$ & $(5.5 \pm 0.5) \cdot 10^{-5}$ \\
\hline isobutanol $\left(\mathrm{CH}_{3}\right)_{2} \mathrm{CHCH}_{2} \mathrm{OH}(\mathrm{I})$ & $167 \pm 5$ & $245 \pm 5$ & $(5.1 \pm 0.7) \cdot 10^{-5}$ \\
\hline ethylene glycol $\mathrm{HOCH}_{2} \mathrm{CH}_{2} \mathrm{OH}$ (I\&I) & $240 \pm 5$ & $\leqslant 250$ & $(3.1 \pm 0.2) \cdot 10^{-5}$ \\
\hline isopropyl alcohol $\mathrm{CH}_{3} \mathrm{CHOHCH}_{3}$ (II) & $138 \pm 5$ & $205 \pm 5$ & $(6.5 \pm 0.7) \cdot 10^{-5}$ \\
\hline tert-butyl alcohol $\mathrm{HOC}\left(\mathrm{CH}_{3}\right)_{3}$ (III) & $140 \pm 5$ & $465-485$ & \\
\hline acetone $\left(\mathrm{CH}_{3}\right)_{2} \mathrm{CO}$ & $120 \pm 5$ & $300-385$ & \\
\hline
\end{tabular}

Table 1: Leidenfrost temperatures, critical reaction temperatures $T_{c}$ and evaporation rates for several chemical compounds. I/II/III refer to primary/secondary/tertiary alcohols respectively. Evaporation rates are obtained from the data of Fig. 2 around a radius $R=2 \mathrm{~mm}$ and assuming spherical droplets: for each chemical compound, the volumetric flow rate is converted into the amount of substance flow rate using the molar mass of the compound and its liquid density at the boiling temperature.

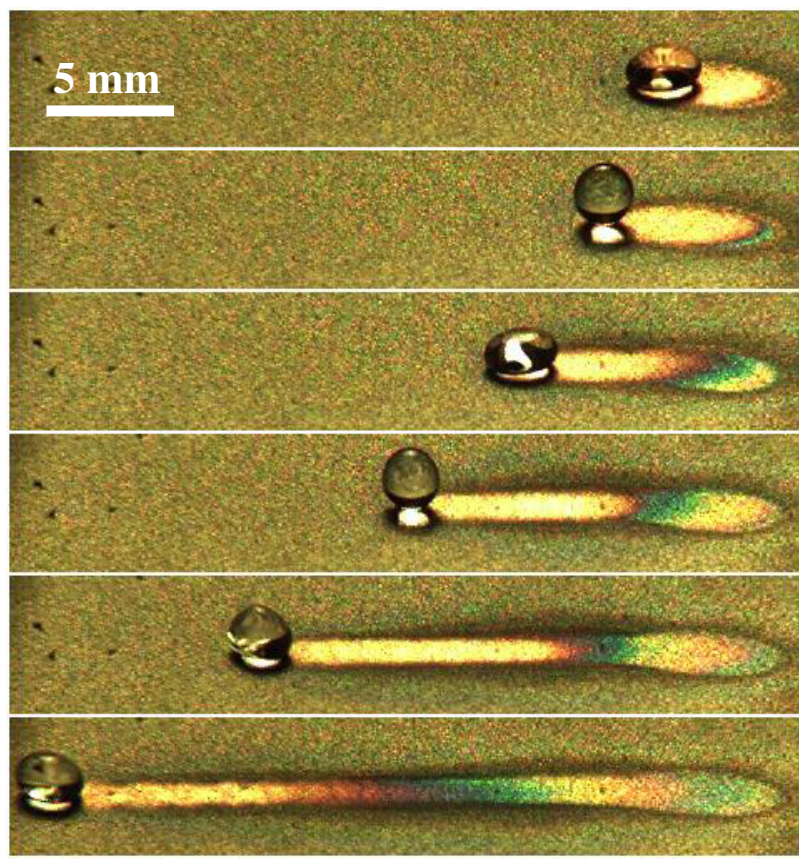

Fig. 4: (Color on-line) Motion of an ethanol Leidenfrost droplet on an inclined plane. The time interval between each picture is of $100 \mathrm{~ms}$. The average sliding velocity between the first and last images is of $50 \mathrm{~mm} / \mathrm{s}$ while the persistence time of the chemical pattern is of a few seconds. heated at $T=450^{\circ} \mathrm{C}$. The inclination of the substrate is of a few degrees and we use a high speed camera at a frame rate of 1000 fps. Six pictures taken during the motion are represented in Fig. 4. The trajectory is given by the native copper tail. This latter is re-oxidized far from the droplet as evidenced by the colored fringes. In the supplementary material we show one movie illustrating the splash of an ethanol droplet (Movie2.avi). The reactive Leindenfrost effect permits to visualize the bouncing of the satellite droplets.

Another application deals with the vapor flow monitoring. Linke et al. have shown that Leidenfrost droplets are self-propelled on ratchet-like substrates in the direction perpendicular to the ratchet features (in the positive

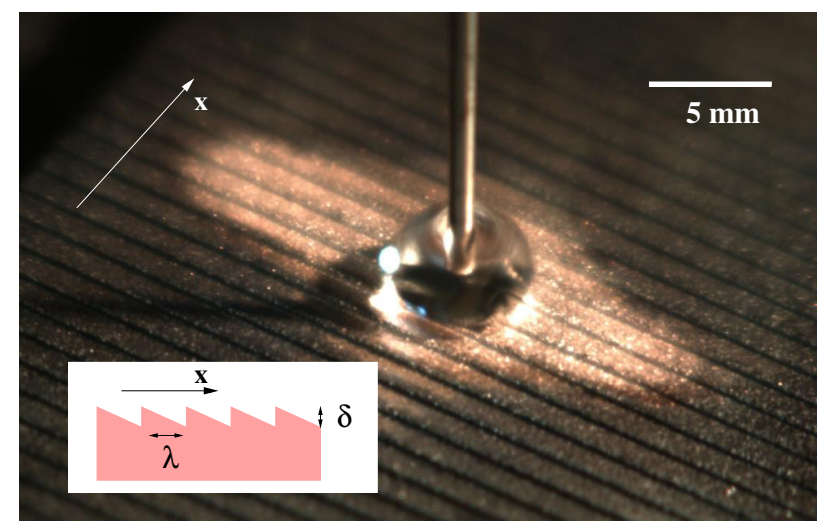

Fig. 5: (Color on-line) Ethanol Leidenfrost droplet standing on a textured copper substrate. The white arrow indicates the direction of the driving force, or equivalently, of the drop motion if the drop is not held by the capillary. Insert : sketch of the ratchet surface.

$x$ direction in Fig. 5) [4]. The origin of the driving force has been intensively studied in the recent years and several theoretical analyses have been proposed. In all of them the role of the rectified vapor flow by the asymmetric teeth of the ratchet is emphasized $[14,15]$. To observe this, we manufactured a textured copper substrate as sketched in the inset of Fig. 5 with the values $\lambda=1.2 \mathrm{~mm}$ and $\delta=0.2$ $\mathrm{mm}$. An ethanol droplet is deposited on the substrate and is maintained by a capillary to prevent its motion. We observe that the native copper spot is not isotropic but extends more significantly in the direction of the ratchet features (perpendicular to the driving force direction $x$ ). This is coherent with the hypothesis that most of the flow is evacuated in the direction perpendicular to the one of the driving force [15].

\section{Conclusion, perspectives. -}

In this article, we have observed and characterized a reactive Leidenfrost effect and some applications. This phenomenon opens a new route to couple thermodynamics, hydrodynamics, capillarity and chemical reactions. In our case it deals with organic liquid droplets standing on a copper substrate. We can easily imagine that such effect 
could occur with any liquid/solid in a Leidenfrost state generating a reactive vapor flow over a reacting substrate.

We thank T. Darmanin and N. Guigo for fruitful discussions.

\section{REFERENCES}

[1] J. G. Leidenfrost, De Aquae Communis Nonnullis Qualitatibus Tractatus, Duisbourg, 1756.

[2] F. Celestini and G. Kirstetter, Effect of the electric field on a Leidenfrost droplet, Soft Matter, 2012, 8, 5992 .

[3] K. Piroird, C. Clanet and D.Quéré, Magnetic control of Leidenfrost drops, Phys. Rev. E, 2012, 85, 056311.

[4] H. Linke, B. J. Aleman, L. D. Melling, M. J. Taormina, M. J. Francis, C. C. Dow-Hygelund, V. Narayanan, R. P. Taylor, and A. Stout, Self-propelled Leidenfrost droplets, Phys. Rev. Lett., 2006, 96, 154502.

[5] T. R. Cousins, R. E. Goldstein, J. W. Jaworski and A. I. Pesci, A ratchet trap for Leidenfrost drops, J. Fluid. Mech., 2012, 79, 215-227.

[6] F. Celestini, T. Frisch and Y. Pomeau, Take-off of small Leidenfrost droplets, Phys. Rev. Lett., 2012, 109, 034501.

[7] A. L. Biance, C. Clanet and D. Quéré, Leidenfrost drops, Phys. Fluids, 2003, 15, 1632, and references therein.

[8] Y. Pomeau, M. Le Berre, F. Celestini and T. Frisch, The Leidenfrost effect: From quasi-spherical droplets to puddles, C. R. Mec., 2012, 340, 867.

[9] R. Abdelaziz, D. Disci-Zayed, M. K. Hedayati, J. H. Pöhls, A. U. Zillohu, B. Erkartal, M. Elbahri, Green chemistry and nanofabrication in a levitated Leidenfrost drop, Nature communications, 2013, 4.

[10] P. H. Boutigny, Études sur les corps à l'état sphéroïdal : Nouvelle branche de physique, 3rd Ed., Paris; Masson, 1857.

[11] C. N. Satterfield, Heterogeneous catalysis in industrial practice, New York, NY (United States); McGraw Hill Book Co., 1991.

[12] J. Adànez, L. F. de Diego, F. García-Labiano, P. Gayán, A. Abad and J. M. Palacios, Selection of oxygen carriers for chemical-looping combustion, Energy Fuels, 2004, 18, 371-377.

[13] B. Sreenivasulu, I. Sreedhar, P. Suresh and K. V. Raghavan, Development trends in porous adsorbents for carbon capture, Environ. Sci. Technol., 2015, 49, 12641-12661.

[14] A. Wurger, Leidenfrost gas ratchets driven by thermal creep, Phys. Rev. Lett., 2011, 107, 164502.

[15] G. Dupeux, M. Le Merrer, G. Lagubeau, C. Clanet, S. Hardt and D. Quéré, Viscous mechanism for Leidenfrost propulsion on a ratchet, Europhys. Lett., 2011, 96, 58001. 